\title{
MicroRNA-199a-5p inhibits VEGF-induced tumorigenesis through targeting oxidored-nitro domain-containing protein 1 in human HepG2 cells
}

\author{
RONG GUI ${ }^{*}$, RONG HUANG ${ }^{*}$, JUN-HUA ZHANG, XIAN-HUI WEN and XIN-MIN NIE \\ The Third Xiangya Hospital, Central South University, Changsha, Hunan 410013, P.R. China
}

Received August 31, 2015; Accepted October 13, 2015

DOI: $10.3892 /$ or.2016.4550

\begin{abstract}
VEGF induces deterioration of hepatocellular carcinoma (HCC) by enhancing cell proliferation and migration. MicroRNAs regulate many cellular processes. In this study, we examined the regulation of tumorigenesis in HCC cells by microRNAs in relation to the effect of VEGF. Differences in microRNA expression between HepG2 and THLE-3 cells were characterized by microarray analysis. The results showed that miR-199a-5p expression was markedly downregulated in HepG2 cells and was inhibited in VEGFoverexpressing HepG2 cells in a dose- and time-dependent manner. This miRNA also inhibited cell proliferation and migration, as demonstrated by MTT and cell migration assays. Oxidored-nitro domain-containing protein $1\left(\mathrm{NOR}_{1}\right)$, a nitroreductase, was identified as a downstream target gene of miR-199a-5p, and upregulation of $\mathrm{NOR}_{1}$ proved critical for the inhibition of VEGF-induced cell proliferation and migration in HepG2 cells by miR-199a-5p. These results indicate that miR-199a-5p is critical for regulating cell proliferation and migration by targeting and upregulating $\mathrm{NOR}_{1}$ in human HepG2 cells.
\end{abstract}

\section{Introduction}

Hepatocellular carcinoma (HCC) is one of the most common and aggressive malignant tumors worldwide and the second leading cause of cancer-related mortality (1). Approximately 10 million HCC patients die every year in China. To date, surgical resection and liver transplantation are the only curative treatment options (2). However, tumor metastasis remains the leading cause of death in HCC patients after resection (3).

Correspondence to: Professor Xin-Min Nie, The Third Xiangya Hospital, Central South University, Changsha, Hunan 410013, P.R. China

E-mail: niexinmin7440@sina.com

${ }^{*}$ Contributed equally

Key words: miR-199a-5p, tumorigenesis, HepG2, $\mathrm{NOR}_{1}$, cell proliferation, cell migration
The proliferation and migration of HCC cells are markedly increased by various growth factors and cytokines such as vascular endothelial growth factor (VEGF) (4), tumor necrosis factor- $\alpha(\mathrm{TNF}-\alpha)(5)$ and granulocyte colony-stimulating factor (G-CSF) (6).

VEGF is one of the most potent stimulants of progression in several tumor types, mainly because it modulates its target transcription factors through multiple signaling pathways $(7,8)$. Indeed, expression of signaling molecules in the VEGF pathway is elevated in several cardiovascular disorders including acute myocardial infarction, coronary artery disease and atherosclerosis (9-11). However, the exact mechanism(s) involved in the process by which VEGF modulates hepatoma cell proliferation and migration remain unclear. Thus, identification of novel molecular mechanisms, particularly novel inhibitors, in the VEGF-dependent hepatoma cell proliferation and migration process has enormous therapeutic potential.

MicroRNAs (miRNAs) are RNA fragments typically composed of only 20-22 nucleotides. They regulate target genes variously at the RNA and/or protein levels and as a result control downstream cellular processes including proliferation, differentiation, and survival (12). Many lines of evidence have implicated miRNAs in modulating hepatoma cell function by targeting the transcriptional factors or signaling molecules involved in tumor cell proliferation and migration such as miR-25, miR-520e and miR-21-3p $(2,13,14)$. Deregulation of miRNAs expression has also been reported in numerous human cancer types, the miRNAs functioning as tumor suppressors in these cases (15). Recently, miR-199a-5p was reported to be an antagonist of tumor cell proliferation. Shi et al revealed that overexpression of miR-199a-5p in porcine preadipocytes significantly promoted cell proliferation while attenuating lipid deposition in subsequent adipocytes (16). Dai et al also demonstrated repression of sustained endoplasmic reticulum stress and hepatocyte apoptosis by endogenous miR-199a-5p by blocking the IRE1 $\alpha$-related pathway (17). Underexpression of miR-199a-5p contributes to the rise of cell invasion in HCC via the functional deregulation of DDR1 activity (18). Hsu et al also showed that upregulation of miR-199a-5p suppresses cell proliferation, motility, and angiogenesis of these ectopic stem cells by targeting the 3'-untranslated region of VEGFA (19).

Here, we found by miRNA microarray expression analysis that miR-199a-5p, which is abundantly expressed in normal 
human liver cells, was significantly downregulated in human hepatoma cells. On this basis, we hypothesized that miR$199 \mathrm{a}-5 \mathrm{p}$ regulates tumorigenesis through some unknown pathway. We then found that miR-199a-5p inhibits human hepatoma cell proliferation and migration through targeting the $\mathrm{NOR}_{1}$ gene, which was previously cloned in our laboratory in the Third Xiang Ya Hospital of Central South University (Hunan, China) and identified as a novel tumor suppressor gene (20).

\section{Materials and methods}

Cell source and culture. The human normal liver (THLE-3) and human hepatoma (HepG2) cell lines were obtained from ATCC. Both cell lines were cultured in RPMI-1640 (Gibco, USA) supplemented with $10 \%$ fetal bovine serum (FBS) (Gibco), $10 \mathrm{mg} / \mathrm{ml}$ streptomycin and $10,000 \mathrm{U} / \mathrm{ml}$ penicillin in a humidified atmosphere of $5 \% \mathrm{CO}_{2}$ at $37^{\circ} \mathrm{C}$. The sequence of miR-199a-5p is ccc agt gtt cag act acc tgt tc, and the antisequence is ggg tca caa gtc tga tgg aca ag.

Microarray analysis. Total RNAs obtained from cells were subjected to the Mammalian miRNA Array Service V4.0 (CapitalBio Corp., Beijing, China) and analyzed.

PCR and quantitative RT-PCR analysis. Total RNAs were extracted using TRIzol reagent (Invitrogen, Carlsbad, CA, USA) following the manufacturer's instructions. PCR was performed for miR-199a-5p (F_Pr: 5'-TCCAGCTGGGCCCAGTGTTCA GACTAC-3';R_Pr:5'-GTGTCGTGGAGTCGGCAATTC-3'), human $\mathrm{NOR}_{1}$ (F_Pr: 5'-TGTTAGGCTAGCGATTGAGTT ATTTGCTTACAC-3' and R_Pr: 5'-CTGCACGAATTCG GTTAAGTATGGCCCGATCTA-3'). SYBR ${ }^{\circledR}$ Green assays (Invitrogen) was used for quantitative RT-PCR. All samples were run in triplicate in 96-well reaction plates using the Applied BioSystems 7300 Sequence Detection system (Perkin-Elmer Applied Biosystems, Foster City, CA, USA). The reactions started at $95^{\circ} \mathrm{C}$ for 5 min followed by 38 cycles of $95^{\circ} \mathrm{C}$ for $15 \mathrm{sec}, 60^{\circ} \mathrm{C}$ for $30 \mathrm{sec}$ and $72^{\circ} \mathrm{C}$ for $30 \mathrm{sec}$. All experiments were repeated three times.

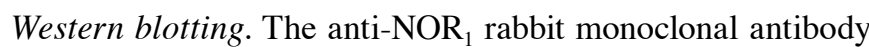
(Epitomics, CA, USA) diluted at 1:1,000, and the anti-GAPDH rat monoclonal antibody (Beyotime, China) at 1:1,000, were used for western blotting. Band intensities were quantified by grayscale and analyzed.

Cell proliferation and migration. Cell proliferation was measured by the MTT assay (MTT cell proliferation assay kit, Invitrogen $\left.{ }^{\mathrm{TM}}\right)$. HepG2 cells $\left(2 \times 10^{4}\right.$ per well) were seeded on cell culture inserts ( $8-\mu \mathrm{m}$ pore size) (Millipore Cell, USA) with serum-free RPMI-1640 in triplicate. Those inserts were then put into a 24-well culture plate. Twelve hours later the medium was removed and the inserts were washed with PBS and stained after fixation. After rinsing with water, images were photographed in three random fields (x400). Cell migration was tested and quantified.

Luciferase reporter assay. The pMIR-NOR -3 '-(UTR) luciferase vector containing the putative binding site for
miR-199a-5p in multiple cloning sites in the 3'-UTR of pMIRREPORT $^{\mathrm{TM}}$ microRNA Expression Reporter Vector (Ambion) was constructed according to the manufacturer's instructions. HepG2 cells were plated at $2 \times 10^{5}$ cells/well in 12 -well plates in triplicate. The pMIR-NOR ${ }_{1}-3^{\prime}-\mathrm{UTR}$ vector $(200 \mathrm{ng})$ together with the $\beta$-gal expressing vector pMIR-REPORT $\beta$-gal (200 ng) (Ambion) were co-transfected with either Pre-miR ${ }^{\mathrm{TM}}$ miRNA precursor molecules or negative control miRNA precursors (Ambion). Luciferase assays and $\beta$-gal enzyme assays were performed $24 \mathrm{~h}$ after the transfection according to the manufacturer's protocol (Promega Corp., Madison, WI, USA). Firefly luciferase activity was normalized to $\beta$-gal expression for each sample.

Construction of lentiviral vector. The lentiviral miR-199a-5p overexpression system (LV-miR-199a-5p) was amplified by PCR. The two ends of miR-199a-5p were linked with restriction enzyme cutting sites. The PCR products of the target gene and pSMPUW-U6-Puro lentiviral expression vector (Cell Biolabs, Inc.) were respectively digested with BamHI and SalI. After purification by electrophoretic separation, the target fragments were induced into competent cells followed by incubation in Luria-Bertani broth containing ampicillin to select overnight. Single colonies were picked as putative products. The products of PCR amplification were identified by restriction endonuclease digestion and sequenced.

Lentiviral packaging and titer determination. The lentiviral vector and pRSV-Rev lentivirus package vector (Invitrogen) were co-transfected into HepG2 cells. Supernatants were collected by centrifugation 24 and 48 h after transfection, respectively, for concentration tests. The lentivirus titers at each time-point were analyzed by the hole-by-dilution titer assay.

Lentiviral transduction. HepG2 cells were infected with lentivirus (LV-miR-199a-5p) when they were 50-70\% confluent. In our pre-experiment, the MOI (multiplicity of infection) value gradients were $0,10,50$ and 100 . Corresponding doses of LV-miR-199a-5p were, respectively, added. LV-GFP was transduced as control. The green fluorescent protein (GFP) expression levels were measured $72 \mathrm{~h}$ after transduction in order to determine the optimal MOI value, which was used in subsequent experiments.

Statistical analysis. All results are presented as means \pm SEM of at least three independent experiments, unless otherwise indicated. Student's t-test was used to assess differences between two groups. $\mathrm{P}<0.05$ was considered statistically significant.

\section{Results}

The expression levels of miRNAs in HepG2 and THLE-3. To identify miRNAs that contribute to the proliferation of liver cells, we performed microarray analysis of miRNA expression in a normal human liver cell line (THLE-3) and a human hepatoma cell line (HepG2). There were significant differences between the two cell lines in the expression of 149 miRNAs, 58 of which were upregulated and 91 down- 
Table I. The expression levels of miRNAs in HepG2 and THLE- 3 cells.

\begin{tabular}{lrr}
\hline miRNA name & score(d) & Fold change \\
\hline miR-199a-5p & -61.3854 & 0.16945 \\
miR-18b & -54.7323 & 0.11342 \\
miR-let-7e & -43.3242 & 0.12435 \\
miR-193a & -36.3287 & 0.08793 \\
miR-224 & -28.6543 & 0.15276 \\
miR-28 & -25.9657 & 0.25738 \\
miR-19a & -20.1985 & 0.09486 \\
miR-434 & -16.9874 & 0.28677 \\
miR-122a & 94.3249 & 28.43587 \\
miR-422b & 85.4535 & 25.45325 \\
miR-520e & 70.45278 & 23.97742 \\
miR-134 & 63.32455 & 14.554325 \\
miR-198 & 43.31445 & 8.43529 \\
miR-202 & 28.45289 & 3.23455 \\
miR-382 & 20.35185 & 2.55325 \\
miR-520b & 13.31457 & 1.98097 \\
\hline
\end{tabular}

regulated. Consistent with previous reports, miR-let-7e was significantly downregulated in the cancer cells (21). miRNAs with significantly altered expression levels are listed in Table I; they include miR-199a-5p, which was significantly downregulated in the hepatoma cells.

miR-199a-5p expression was attenuated in VEGF-induced HepG2 cells. VEGF contributes to some key stages of tumorigenesis, including the function of cancer stem cells and tumor initiation (22). To confirm the importance of miR-199a-5p in regulating tumorigenesis, we performed miRNA microarray analysis in HepG2 cells at 0,3,6, and $24 \mathrm{~h}$ after VEGF stimulation with an $\mathrm{IC}_{50}$ of $60 \mathrm{ng} / \mathrm{ml}$ (Fig. 1B).
The relative expression levels of miR-199a-5p were normalized to U6, the endogenous control. The results showed that VEGF stimulation on HepG2 cells caused a significant dose-dependent reduction in the expression levels of miR-199a (Fig. 1A). These findings revealed that miR-199a and VEGF expressions are negatively correlated.

Effects of micro-RNA199a-5p and VEGF on HepG2 proliferation. To examine the effects of microRNA199a-5p in HepG2 cells further, we examined whether its restoration would affect VEGF-induced cell proliferation. We constructed a lentiviral vector expressing miR-199a-5p (LV-miR-199a-5p) and showed that transductions with increasing multiplicity of infections (MOI) of LV-miR-199a-5p significantly elevated miR-199a-5p expression in HepG2 cells (Fig. 2A). Forced expression of miR-199a-5p by LV-miR-199a-5p strongly inhibited cell proliferation over the range 10-100 MOI (Fig. 2B). The effects of miR-199a-5p on HepG2 cell proliferation were further elucidated by measuring the expression of PCNA, a marker of tumor cell proliferation. VEGF significantly increased PCNA expression, but this was markedly attenuated in miR-199a-5poverexpressing cells (Fig. 2C and D), while miR-199a-5p knock-down by anti-miR-199a-5p increased proliferation (Fig. 2E and F).

Effects of micro-RNA199a and VEGF on hepatoma cell migration. Hepatoma cell migration is also thought to make an important contribution to the progression of HCC. To determine whether miR-199a-5p is also involved in this process, we performed cell migration experiments, and the results verified that miR-199a-5p suppresses tumorigenesis. miR-199a inhibited cell migration induced by VEGF overexpression (Fig. 3A and B). Moreover, consistent with our hypothesis, the inhibition of miR-199a expression by anti-miR-199a restored the capacity of HepG2 cells for migration (Fig. 3C and D). These results suggest that miR-199a modulates tumorigenesis by changing key factors in the tumor microenvironment.

$N O R_{l}$ is a direct target of miR-199a-5p. Our previous study revealed $\mathrm{NOR}_{1}$ as a candidate tumor suppressor that inhibits
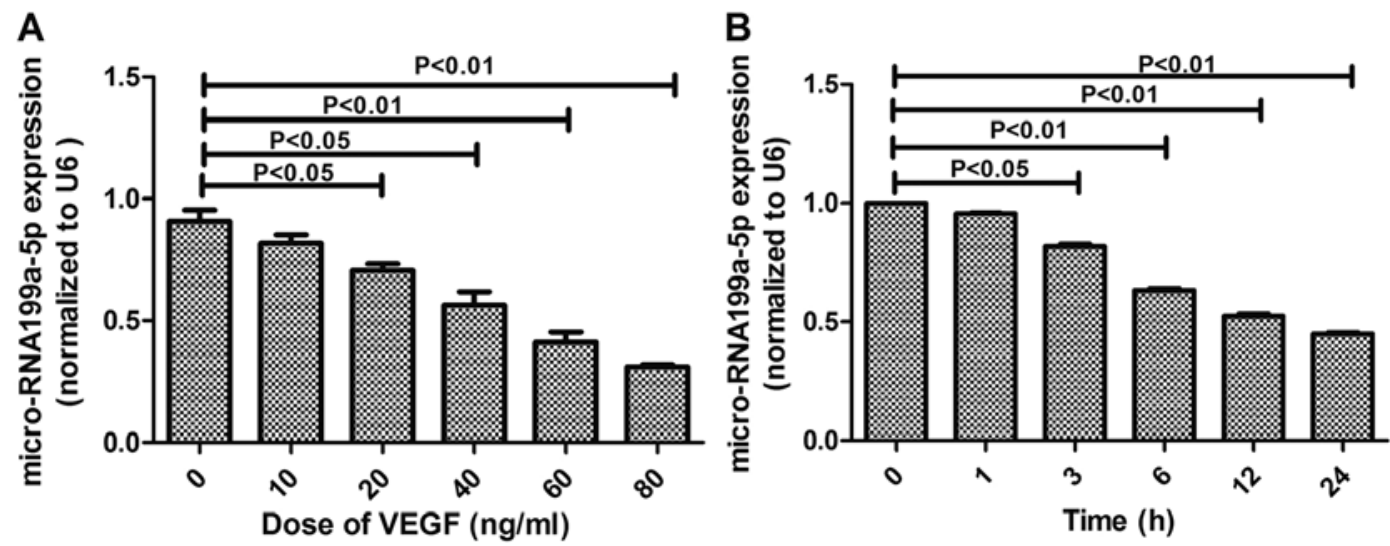

Figure 1. miR-199a-5p expression is attenuated in VEGF-induced HepG2 cells. HepG2 cells, seeded in 6-well plates, were cultured with 0.5\% FBS for 48 h before treatment with either VEGF $(60 \mathrm{ng} / \mathrm{ml})$ or RPMI-1640 as a negative control. (A) VEGF caused a dose-dependent decrease of miR-199a-5p expression in HepG2 cells in $24 \mathrm{~h}$, which was tested by qRT-PCR $(\mathrm{n}=3)(\mathrm{P}<0.05)$. (B) VEGF caused a time-dependent decrease of miR-199a expression in HepG2 cells, detected by qRT-PCR $(n=3)(P<0.05)$. 

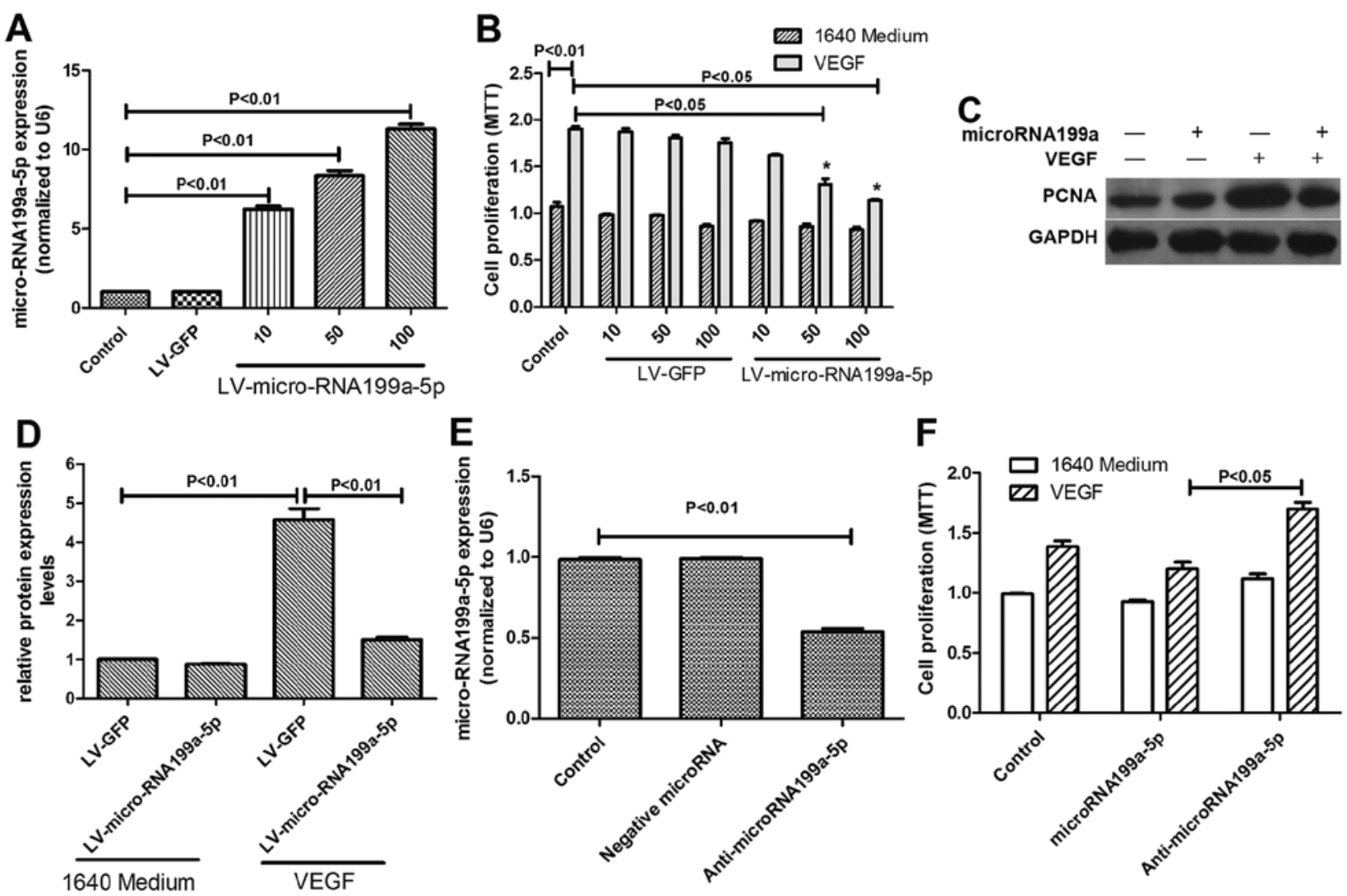

Figure 2. miR-199a-5p inhibits VEGF-induced HepG2 proliferation. (A) HepG2 cells were infected with LV-miR-199a-5p or LV-GFP at different MOI LV-miR-199a-5p induced the overexpression of miR-199a-5p in a dose-dependent manner. (B) HepG2 cells were infected with LV-miR-199a-5p (0-100 MOI) or LV-GFP (0-100 MOI), treated with or without VEGF $(60 \mathrm{ng} / \mathrm{ml})$ for $24 \mathrm{~h} . \mathrm{LV}-\mathrm{miR}-199 \mathrm{a}-5 \mathrm{p}$ significantly decreased VEGF-induced cell proliferation, shown by the MTT proliferation assay (n=3) P<0.05. (C) HepG2 cells infected with LV-miR-199a-5p (100 MOI) or LV-GFP (100 MOI) were incubated with VEGF $(60 \mathrm{ng} / \mathrm{ml})$ for $24 \mathrm{~h}$ and analyzed for PCNA protein levels by western blotting. (D) PCNA protein levels in the western blots were quantified by grayscale and analyzed $(\mathrm{n}=3)$. (E) The inhibitory effect of anti-microRNA199a-5p on miR-199a-5p expression in HepG2 cells. (F) Anti-miR-199a-5p (80 nM) significantly restored HepG2 cell proliferation stimulated by VEGF $(60 \mathrm{ng} / \mathrm{ml})$, detected by the MTT proliferation assay $(\mathrm{n}=3)$.

A
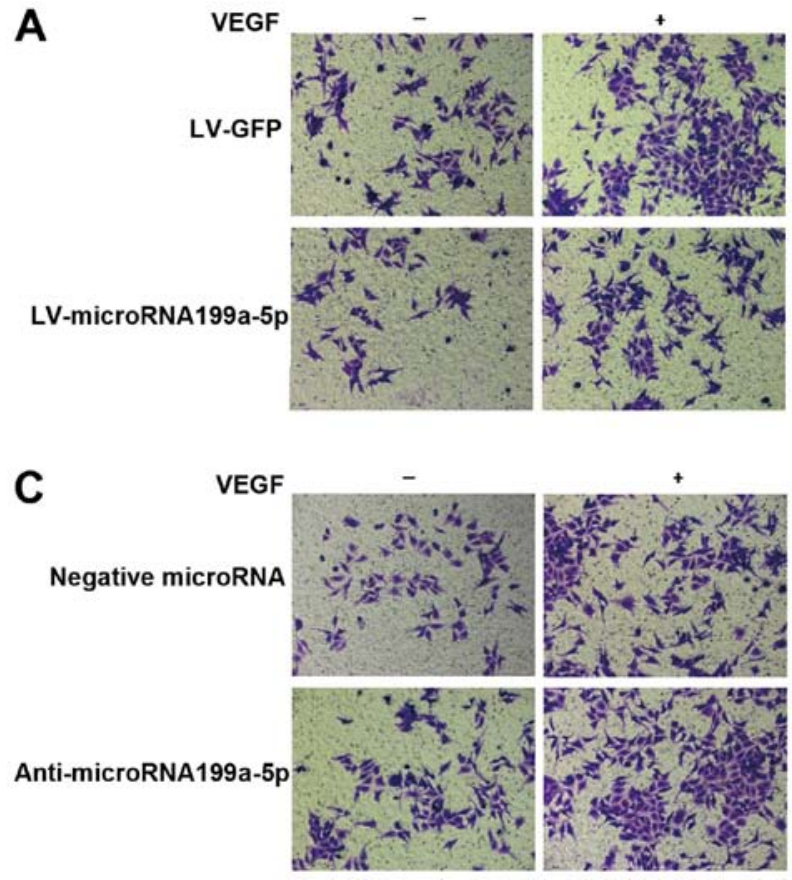
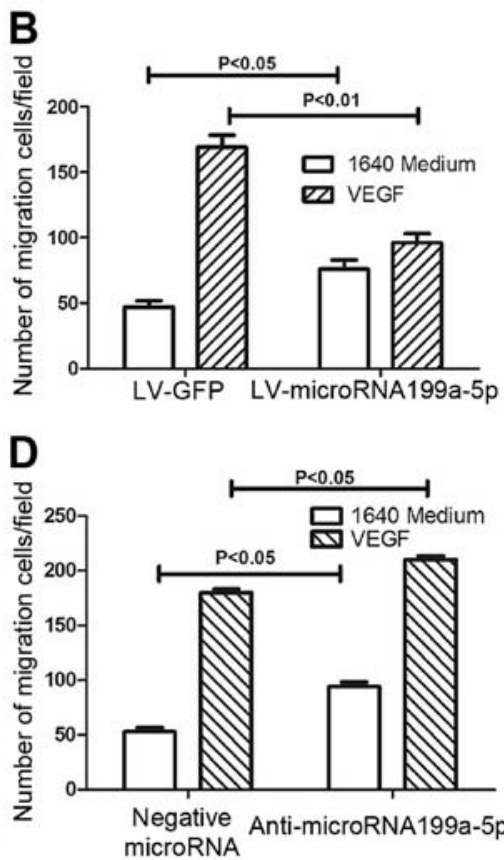

Figure 3. miR-199a-5p inhibits VEGF-induced hepatoma cell migration. (A) HepG2 cells were seeded in triplicate on inserts and incubated for $12 \mathrm{~h}$ with or without either VEGF or LV-micro-RNA199a before staining. (B) The number of migrated cells was quantified (x400). Results were confirmed by triple independent experiments. (C) After infection with anti-miR-199a-5p or negative control oligos, HepG2 cells were seeded in triplicate on inserts and incubated for $12 \mathrm{~h}$ with or without VEGF; migrated cells were counted (x400). (D) The number of migrated cells was quantified (x400). Results from three independent experiments are reported. 


\section{A NOR $_{1}-3$ 'URT 5 '....CCGGGGCTCTTCACAAGTCCAGCTCGTTTC... \\ microRNA-199a-5p \\ CCCAGTGTTCAGACTACCTGTTC}

NOR $_{1}$-3'URT 2 5'....CCGGGGCTCTTGGGCAAGCCAGCTCGTTTC...
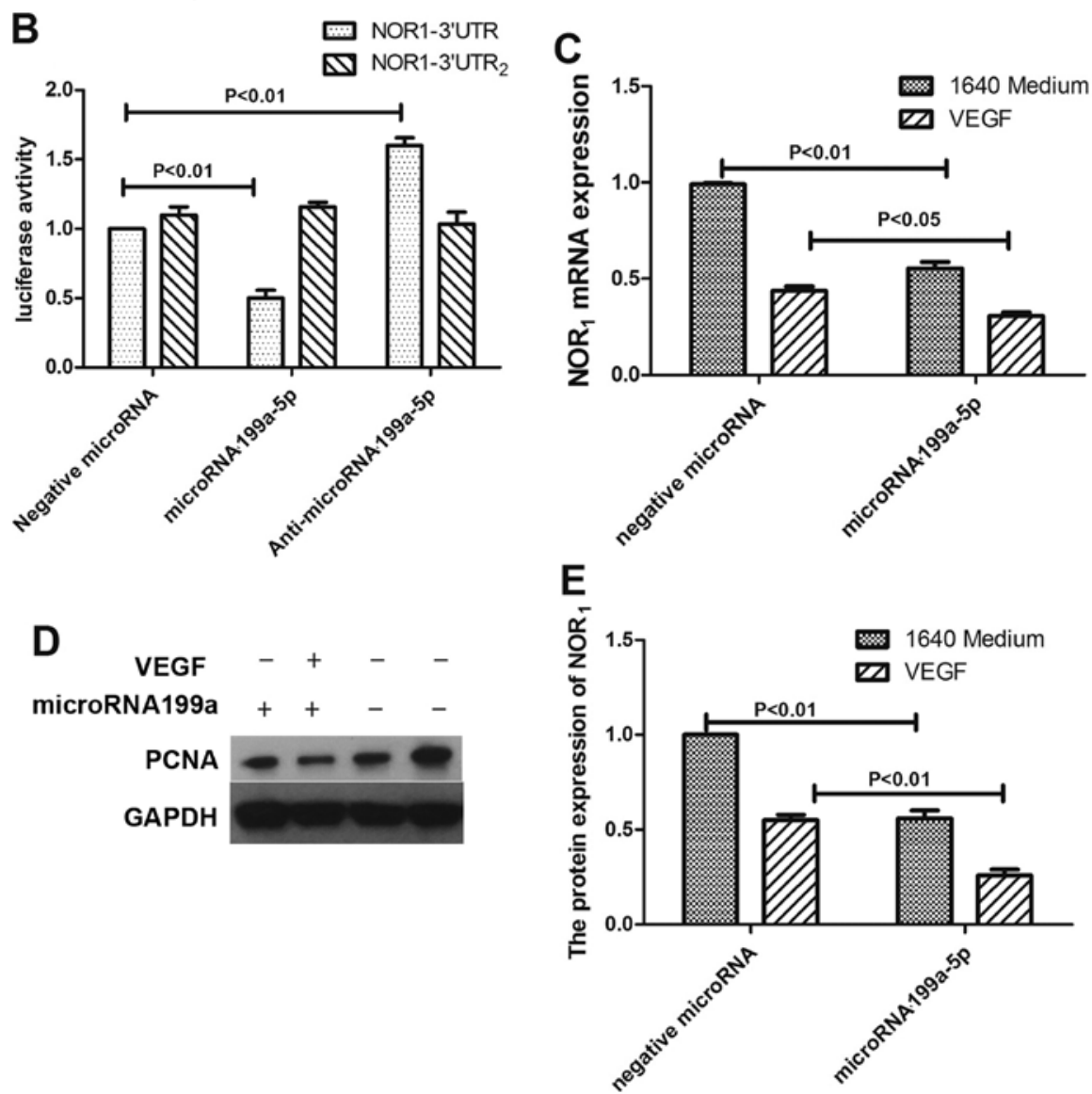

Figure 4. NOR ${ }_{1}$ is a direct target of miR-199a-5p. (A) The sequences of wild-type and mutated NOR $3^{\prime}-\mathrm{UTR}\left(\mathrm{NOR}_{1} 3^{\prime}-\mathrm{UTR}^{\prime}\right.$ and NOR $3^{\prime}-\mathrm{UTR}_{2}$, respectively) binding sites for miR-199a-5p are shown. (B) HepG2 cells were co-transfected by the luciferase reporter vectors carrying either NOR $3^{3}$-UTR or NOR $33^{\prime}-\mathrm{UTR}^{-}$ with miR-199a-5p, anti-miR-199a-5p, or negative microRNA. Luciferase was assayed $48 \mathrm{~h}$ after transfection. (C) miR-199a-5p or negative microRNAs were

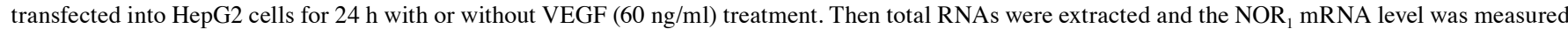

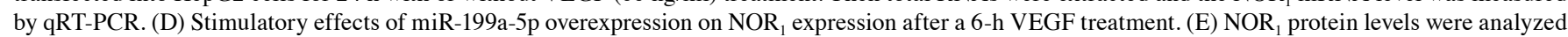
by grayscale.

the development and/or progression of tumors (23). In this study, when we searched the target scan database, we found that $\mathrm{NOR}_{1}$ is a potential target of miR-199a-5p. As shown in Fig. 4A, human $\mathrm{NOR}_{1}$ mRNA has a potential miR-199a-5p binding site in its 3'-UTR. To verify whether miR-199a-5p binds directly to the 3'-UTR sequence of $\mathrm{NOR}_{1}$ mRNA, downregulating its expression, the 3 '-UTR sequence containing the putative binding was cloned into an assay-ready luc-UTR reporter vector. The constructed vector was then co-transfected with either miR-199a-5p or control plasmid into HepG2 cells. As shown in Fig. 4B, the luciferase activity was inhibited in the miR-199a-5p-induced cells but not in the control group. To verify that $\mathrm{NOR}_{1}$ is a functional target gene of miR-199a-5p, we induced miR-199a-5p into HepG2 cells and found that $\mathrm{NOR}_{1}$ expression was decreased at both the mRNA and protein levels despite VEGF treatment (Fig. 4C-E). We therefore measured the proliferation and migration of HepG2 cells treated with $\mathrm{NOR}_{1}$ to test its role in the inhibition of tumorigenesis by miR- 199a-5p. The results indicate that overexpression of $\mathrm{NOR}_{1}$ significantly inhibits the proliferation (Fig. 5A) and migration (Fig. 5B and C) of HepG2 cells.

\section{Discussion}

miR-199a-5p has been shown to have a wide range of functions and can behave differently in different systems and diseases. For example, it is a major regulator of lung fibroblasts and livers in injured tissues $(24,25)$. Rapid downregulation of miR-199a-5p is required for upregulation of HIF1 $\alpha$ to protect cells exposed to hypoxia (26). Its behavior in tumors could be more complicated. In different contexts it can function either as an oncogene $(27,28)$ or as a tumor suppressor $(29,30)$. In our previous study, miR-199a-5p was confirmed as a tumor suppressor in hepatocellular carcinoma. However, details of the mechanism by which it regulates tumorigenesis remained unclear. In our present study, we demonstrated that it inhibits 


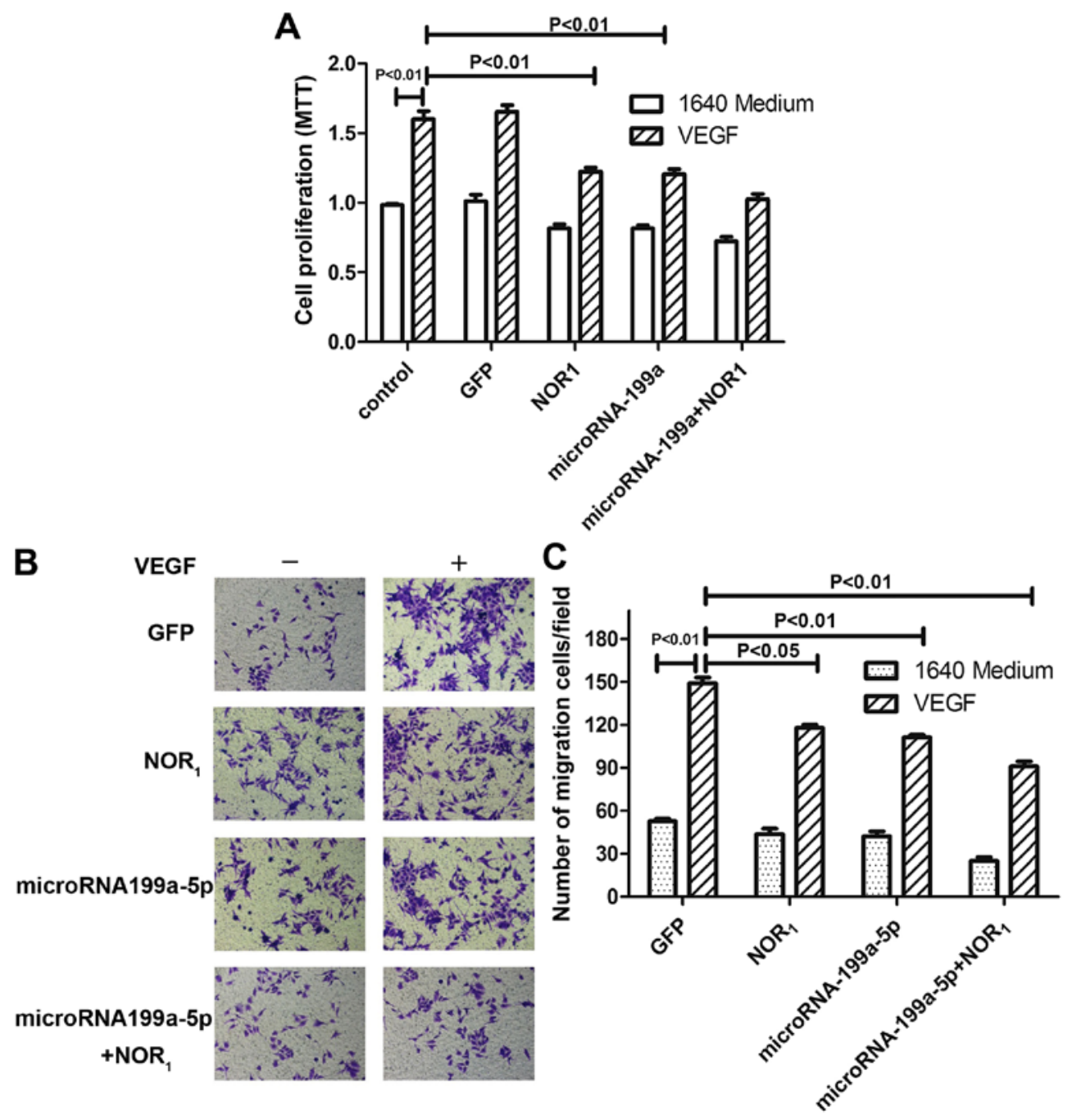

Figure 5. miR-199a-5p inhibits cell proliferation and migration through targeting NOR . $_{1}$ (A) HepG2 cells were infected with either GFP, NOR 1 , miR-199a-5p or miR-199a-5p supplemented with $N_{1}$, treated with or without VEGF $(60 \mathrm{ng} / \mathrm{ml})$. The cell proliferation levels were tested by the MTT method, $\mathrm{n}=3(\mathrm{P}<0.05)$. (B) HepG2 cells were infected as in (A) and transferred into cell inserts at $2 \times 10^{4}$ cells/insert, and then cell migration was measured after stimulation by VEGF for $24 \mathrm{~h}, \mathrm{n}=3$. (C) The number of migrating cells was quantified (x400). Results from three independent experiments are reported.

HepG2 cell proliferation and migration through targeting $\mathrm{NOR}_{1}$. We also found that miR-199a-5p expression in hepatocellular carcinoma was markedly lower than in normal liver cells because it was downregulated both time- and dose-dependently by VEGF stimulation, which induces the deterioration of hepatocellular carcinoma. Restoration of miR-199a-5p markedly attenuated VEGF-induced HepG2 cell proliferation and migration, and knock-down of miR199a-5p by anti-miR-199a-5p had the opposite effect on tumorigenesis.

MicroRNAs are highly conserved small regulatory RNAs that antagonize the expression of target genes by hybridizing to specific binding sites in the 3'-untranslated regions (UTR) of many mRNAs (31). Upon microRNA-guided recruitment of a multi-protein complex, either the target mRNA is degraded directly or its translation is blocked, depending on the complementarity between the microRNA and its binding site (32). In our study, we confirmed that miR-199a-5p inhibited cell proliferation and migration via $\mathrm{NOR}_{1}$ by binding directly to the 3'-UTR of its mRNA. NOR ${ }_{1}$, the gene for which is located in a $120 \mathrm{~kb}$ region at $1 \mathrm{p} 34.3$, was first isolated from naso- pharyngeal cells (NPCs) (23). Zeng et al showed that $\mathrm{NOR}_{1}$ mRNA expression was frequently downregulated in NPCs (33). This epigenetic silencing of $\mathrm{NOR}_{1}$ impaired the cellular protective response to environmental stresses by normal NPCs and promoted nasopharyngeal carcinogenesis (34). Our previous study confirmed $\mathrm{NOR}_{1}$ as a tumor suppressor, the expression of which was significantly downregulated in nasopharyngeal carcinoma, hepatocellular carcinoma and gastric cancer (20). Previously, regulation of $\mathrm{NOR}_{1}$ expression by microRNAs had not been demonstrated. In this study, we showed that miR-199a-5p binds directly to the 3'-UTR of human $\mathrm{NOR}_{1}$ mRNA and downregulates $\mathrm{NOR}_{1}$ expression at both the mRNA and protein levels with or without VEGF stimulation. Overexpression of $\mathrm{NOR}_{1}$ strongly inhibited the proliferation and migration of HepG2 cells, i.e., miR-199a-5p is a novel post-transcriptional regulator of $\mathrm{NOR}_{1}$.

In conclusion, this study identified miR-199a-5p as a potential regulator of human HepG2 cells that acts by targeting the 3'-UTR of $\mathrm{NOR}_{1}$ mRNA. miR-199a-5p expression was substantially downregulated in human hepatocellular carcinoma but not in normal liver cells. Re-establishment 
of its expression markedly inhibited both cell proliferation and migration in response to VEGF stimulation. Our study provides novel insight into the molecular mechanisms associated with tumorigenesis, and suggests a potential therapeutic target for human HCC treatment.

In conclusion, this study showed that miR-199a-5p antagonizes tumorigenesis owing to its inhibitory role in cell proliferation and migration. Through binding to the 3'-UTR of human $\mathrm{NOR}_{1}$ mRNA, miR-199a-5p enhances NOR N $_{1}$ expression, and then $\mathrm{NOR}_{1}$ inhibits cell proliferation and migration induced by VEGF.

\section{Acknowledgements}

This study was supported by National Natural Science Foundation of China (NSFC) (grant nos. 81573091, 30300383, 81072270 and 81101828), and Fundamental Research Funds for the Central Universities (no. 2011JQ030).

\section{References}

1. Jemal A, Bray F, Center MM, Ferlay J, Ward E and Forman D: Global cancer statistics. CA Cancer J Clin 61: 69-90, 2011.

2. Su ZX, Zhao J, Rong ZH, Geng WM, Wu YG and Qin CK: Upregulation of microRNA-25 associates with prognosis in hepatocellular carcinoma. Diagn Pathol 9: 47, 2014.

3. Li S, Dong P, Wang J, Zhang J, Gu J, Wu X, Wu W, Fei X, Zhang Z, Wang Y, et al: Icariin, a natural flavonol glycoside, induces apoptosis in human hepatoma SMMC-7721 cells via a ROS/JNK-dependent mitochondrial pathway. Cancer Lett 298 : 222-230, 2010

4. Zhan P, Qian Q and Yu LK: Serum VEGF level is associated with the outcome of patients with hepatocellular carcinoma: A meta-analysis. Hepatobiliary Surg Nutr 2: 209-215, 2013.

5. Cao X, Zhang L, Shi Y, Sun Y, Dai S, Guo C, Zhu F, Wang Q, Wang J, Wang X, et al: Human tumor necrosis factor (TNF)alpha-induced protein 8-like 2 suppresses hepatocellular carcinoma metastasis through inhibiting Rac1. Mol Cancer 12: 149,2013

6. Miyahara K, Nouso K, Morimoto Y, Takeuchi Y, Hagihara H, Kuwaki K, Onishi H, Ikeda F, Miyake Y, Nakamura S, et al; Okayama Liver Cancer Group: Pro-angiogenic cytokines for prediction of outcomes in patients with advanced hepatocellular carcinoma. Br J Cancer 109: 2072-2078, 2013

7. Duong T, Koltowska K, Pichol-Thievend C, Le Guen L, Fontaine F, Smith KA, Truong V, Skoczylas R, Stacker SA, Achen MG, et al: VEGFD regulates blood vascular development by modulating SOX18 activity. Blood 123: 1102-1112, 2014.

8. Ryzhov S, Biktasova A, Goldstein AE, Zhang Q, Biaggioni I, Dikov MM and Feoktistov I: Role of JunB in adenosine A2B receptor-mediated vascular endothelial growth factor production. Mol Pharmacol 85: 62-73, 2014.

9. Yao HC, Liu T, Meng XY, Han QF, Zhang M and Wang LX: Effect of basic fibroblast growth factor on the myocardial expression of hypoxia-inducible factor- $1 \alpha$ and vascular endothelial growth factor following acute myocardial infarction. Heart Lung Circ 22: 946-951, 2013.

10. Cui QT, Li Y, Duan CH, Zhang W and Guo XL: Further evidence for the contribution of the vascular endothelial growth factor gene in coronary artery disease susceptibility. Gene 521: 217-221, 2013.

11. Winnik S, Lohmann C, Siciliani G, von Lukowicz T, Kuschnerus K, Kraenkel N, Brokopp CE, Enseleit F, Michels S, Ruschitzka F, et al: Systemic VEGF inhibition accelerates experimental atherosclerosis and disrupts endothelial homeostasis - implications for cardiovascular safety. Int J Cardiol 168: 2453-2461, 2013

12. Ambros V: The functions of animal microRNAs. Nature 431: 350-355, 2004

13. Lo TF, Tsai WC and Chen ST: MicroRNA-21-3p, a berberineinduced miRNA, directly down-regulates human methionine adenosyltransferases $2 \mathrm{~A}$ and $2 \mathrm{~B}$ and inhibits hepatoma cell growth. PLoS One 8: e75628, 2013.
14. Li BA: A novel tumor suppressor miRNA miR-520e contributes to suppression of hepatoma. Acta Pharmacol Sin 33: 3-4, 2012.

15. Iorio MV and Croce CM: microRNA involvement in human cancer. Carcinogenesis 33: 1126-1133, 2012.

16. Shi XE, Li YF, Jia L, Ji HL, Song ZY, Cheng J, Wu GF, Song CC, Zhang QL, Zhu JY, et al: MicroRNA-199a-5p affects porcine preadipocyte proliferation and differentiation. Int J Mol Sci 15: 8526-8538, 2014

17. Dai BH, Geng L, Wang Y, Sui CJ, Xie F, Shen RX, Shen WF and Yang JM: microRNA-199a-5p protects hepatocytes from bile acid-induced sustained endoplasmic reticulum stress. Cell Death Dis 4: e604, 2013.

18. Shen Q, Cicinnati VR, Zhang X, Iacob S, Weber F, Sotiropoulos GC, Radtke A, Lu M, Paul A, Gerken G, et al: Role of microRNA-199a-5p and discoidin domain receptor 1 in human hepatocellular carcinoma invasion. Mol Cancer 9: 227, 2010.

19. Hsu CY, Hsieh TH, Tsai CF, Tsai HP, Chen HS, Chang Y, Chuang HY, Lee JN, Hsu YL and Tsai EM: miRNA-199a-5p regulates VEGFA in endometrial mesenchymal stem cells and contributes to the pathogenesis of endometriosis. J Pathol 232: 330-343, 2014.

20. Gui R, Li D, Qi G, Suhad A and Nie X: Inhibition of Grb2mediated activation of MAPK signal transduction suppresses NOR1/CB1954-induced cytotoxicity in the HepG2 cell line. Oncol Lett 4: 566-570, 2012.

21. Cai J, Yang C, Yang Q, Ding H, Jia J, Guo J, Wang J and Wang Z: Deregulation of let-7e in epithelial ovarian cancer promotes the development of resistance to cisplatin. Oncogenesis 2: e75, 2013

22. Goel HL and Mercurio AM: VEGF targets the tumour cell. Nat Rev Cancer 13: 871-882, 2013.

23. Nie X, Zhang B, Li X, Xiang J, Xiao B, Ma J, Zhou M, Zhu S, $\mathrm{Lu} \mathrm{H}$, Gui R, et al: Cloning, expression, and mutation analysis of NOR1, a novel human gene down-regulated in HNE1 nasopharyngeal carcinoma cell line. J Cancer Res Clin Oncol 129: 410-414, 2003.

24. Lino Cardenas CL, Henaoui IS, Courcot E, Roderburg C, Cauffiez C, Aubert S, Copin MC, Wallaert B, Glowacki F, Dewaeles E, et al: miR-199a-5p Is upregulated during fibrogenic response to tissue injury and mediates TGFbeta-induced lung fibroblast activation by targeting caveolin-1. PLoS Genet 9: e1003291, 2013.

25. Ogawa T, Enomoto M, Fujii H, Sekiya Y, Yoshizato K, Ikeda K and Kawada N: MicroRNA-221/222 upregulation indicates the activation of stellate cells and the progression of liver fibrosis. Gut 61: 1600-1609, 2012.

26. Sayed D and Abdellatif M: AKT-ing via microRNA. Cell Cycle 9: 3213-3217, 2010

27. Magrelli A, Azzalin G, Salvatore M, Viganotti M, Tosto F, Colombo T, Devito R, Di Masi A, Antoccia A, Lorenzetti S, et al: Altered microRNA expression patterns in hepatoblastoma patients. Transl Oncol 2: 157-163, 2009.

28. Pencheva N, Tran H, Buss C, Huh D, Drobnjak M, Busam K and Tavazoie SF: Convergent multi-miRNA targeting of ApoE drives LRP1/LRP8-dependent melanoma metastasis and angiogenesis. Cell 151: 1068-1082, 2012.

29. Cheung HH, Davis AJ, Lee TL, Pang AL, Nagrani S, Rennert OM and Chan WY: Methylation of an intronic region regulates miR-199a in testicular tumor malignancy. Oncogene 30: 3404-3415, 2011

30. Wang F, Zheng Z, Guo J and Ding X: Correlation and quantitation of microRNA aberrant expression in tissues and sera from patients with breast tumor. Gynecol Oncol 119: 586-593, 2010.

31. Augustin R, Endres K, Reinhardt S, Kuhn PH, Lichtenthaler SF, Hansen J, Wurst W and Trümbach D: Computational identification and experimental validation of microRNAs binding to the Alzheimer-related gene ADAM10. BMC Med Genet 13: 35, 2012.

32. Scherr M, Venturini L and Eder M: Lentiviral vector-mediated expression of pre-miRNAs and antagomiRs. Methods Mol Biol 614: 175-185, 2010

33. Zeng Z, Zhou Y, Xiong W, Luo X, Zhang W, Li X, Fan S, Cao L, Tang K, Wu M, et al: Analysis of gene expression identifies candidate molecular markers in nasopharyngeal carcinoma using microdissection and cDNA microarray. J Cancer Res Clin Oncol 133: 71-81, 2007.

34. Li W, Li X, Wang W, Li X, Tan Y, Yi M, Yang J, McCarthy JB, Xiong W, Wu M, et al: NOR1 is an HSF1- and NRF1-regulated putative tumor suppressor inactivated by promoter hypermethylation in nasopharyngeal carcinoma. Carcinogenesis 32: 1305-1314, 2011 